\title{
T-CONVERGENCE AND ABSOLUTE MINIMIZERS FOR SUPREMAL FUNCTIONALS
}

\author{
Thierry Champion ${ }^{1}$, Luigi De Pascale ${ }^{2}$ and Francesca Prinari ${ }^{3}$
}

\begin{abstract}
In this paper, we prove that the $L^{p}$ approximants naturally associated to a supremal functional $\Gamma$-converge to it. This yields a lower semicontinuity result for supremal functionals whose supremand satisfy weak coercivity assumptions as well as a generalized Jensen inequality. The existence of minimizers for variational problems involving such functionals (together with a Dirichlet condition) then easily follows. In the scalar case we show the existence of at least one absolute minimizer (i.e. local solution) among these minimizers. We provide two different proofs of this fact relying on different assumptions and techniques.
\end{abstract}

Mathematics Subject Classification. 49J45, 49J99.

Received September 26, 2002. Revised April 8, 2003.

\section{INTRODUCTION}

The classical problems of Calculus of Variations are concerned with the minimization of integral functionals. From the point of view of modelization, this usually corresponds to minimizing the mean of a certain pointwise quantity which can be an energy density or a cost for unitary work. In other words, minimizing the functional is equivalent to controlling the mean of some quantity. On the other hand there are situations in which a control on the worst (or best) situation is needed, in the sense that one wants to control the maximum of a certain quantity instead of its mean. This last type of problems can be easily formulated as a problem of Calculus of Variations or Optimal Control Theory in $L^{\infty}$, and it received a lot of attention in the last ten years.

The problems of Calculus of Variations in $L^{\infty}$ that we will study in this paper are formulated as follows:

$$
\min _{v \in W_{g}^{1, \infty}\left(\Omega, \mathbb{R}^{M}\right)}\{F(v):=\underset{x \in \Omega}{\operatorname{ess} . \sup } f(x, v(x), D v(x))\},
$$

where $\Omega$ is a bounded open subset of $\mathbb{R}^{N}, f$ satisfies the natural assumption to have the measurability of $f(x, v(x), D v(x))$ for all $v \in W^{1, \infty}\left(\Omega, \mathbb{R}^{M}\right), g$ is a map in $W^{1, \infty}\left(\Omega, \mathbb{R}^{M}\right) \cap \mathrm{C}\left(\bar{\Omega}, \mathbb{R}^{M}\right)$ and $W_{g}^{1, \infty}\left(\Omega, \mathbb{R}^{M}\right)$ denotes the space of functions such that $(u-g) \in W^{1, \infty}\left(\Omega, \mathbb{R}^{M}\right) \cap \mathrm{C}_{0}\left(\Omega, \mathbb{R}^{M}\right)$. According to part of the already existing literature we will refer to functionals of that type as supremal functionals while we will refer to $f$ as supremand.

\footnotetext{
Keywords and phrases. Supremal functionals, lower semicontinuity, generalized Jensen inequality, absolute minimizer (AML, local minimizer), $L^{p}$ approximation.

${ }^{1}$ Laboratoire d'Analyse Non Linéaire Appliquée, U.F.R. des Sciences et Techniques, Université de Toulon et du Var, Avenue de l'Université, BP. 132, 83957 La Garde Cedex, France; e-mail: champion@unic.tln.fr

2 Dipartimento di Matematica Applicata, Universitá di Pisa, Via Bonanno Pisano 25/B, 56126 Pisa, Italy.

${ }^{3}$ Dipartimento di Matematica, Universitá di Pisa, Via Buonarroti 2,56127 Pisa, Italy.
} 
Two relevant facts distinguish the classical integral problems of the Calculus of Variations from problems involving a supremal functional. The first one is that when one works with a supremal functional, sets of arbitrarily small measure can not be neglected. The second one is that the problem (1.1) is in some sense non-local. By this last sentence we mean that modifying a function $v$ on a set of positive measure may not change the value of the functional $F(v)$. The first fact plays a role in studying the semicontinuity and relaxation of supremal functionals, while the second come into play in studying minimizers.

Both differences are due to the fact that integral functionals are additive while supremal functionals are only subadditive with respect to the union of sets. The additivity property of integral functionals implies that a minimizer $u$ for an integral functional $G$ defined on some space of measurable functions $X$ by:

$$
G(v):=\int_{\Omega} g(x, v(x), D v(x)) \mathrm{d} x
$$

is always a local minimizer, which means that for all subdomain $V \subset \Omega$ one has

$$
\int_{V} g(x, u(x), D u(x)) \mathrm{d} x \leq \int_{V} g(x, v(x), D v(x)) \mathrm{d} x
$$

whenever $v \in X$ with $v=u$ on $\partial V$. By analogy with the case of integral functionals, reference [2] defined the following notion of local minimizers for supremal functionals.

Definition 1.1. A local minimizer for (1.1) is a minimizer $u \in W^{1, \infty}\left(\Omega, \mathbb{R}^{M}\right) \cap \mathrm{C}\left(\bar{\Omega}, \mathbb{R}^{M}\right)$ such that for all open subset $V \subset \Omega$ one has

$$
\underset{x \in V}{\operatorname{ess} . \sup } f(x, u(x), D u(x)) \leq \underset{x \in V}{\operatorname{ess.sup}} f(x, v(x), D v(x))
$$

for all $v$ in $W^{1, \infty}\left(V, \mathbb{R}^{M}\right) \cap \mathrm{C}\left(\bar{V}, \mathbb{R}^{M}\right)$ such that $v=u$ on $\partial V$. In the following, a local minimizer will be called an AML.

The name AML stands for Absolute Minimizing Lipschitz and it was originally referred to the scalar case where $f(x, s, A)=|A|$. Before going further, we notice that a minimizer for a supremal functional is not necessarily a local minimizer.

Example 1.2. Consider the following variational problem on $\Omega:=]-2,2[$ :

$$
\min \left\{\underset{x \in]-2,2[}{\operatorname{ess} . \sup } f\left(x, v^{\prime}(x)\right): v(-2)=v(2)=0\right\}
$$

where the supremand $f$ is given by

$$
f(x, s):= \begin{cases}1+|s-1| & \text { if } x \in]-2,-1[\cup] 1,2[, \\ |s| / 2 & \text { if } x \in[-1,1]\end{cases}
$$

The set of optimal solutions of (1.2) is the set of Lipschitz continuous functions $u$ such that: $u(x)=x+2$ for $x \in[-2,-1], u(x)=x-2$ for $x \in[1,2]$ and $u$ has Lipschitz constant less that 2 on $[-1,1]$. An AML of this problem should be an optimal solution of the localized problem on $V=]-1,1[$, which then reads:

$$
\min \left\{\operatorname{ess.sup}_{x \in]-1,1[}\left|v^{\prime}(x)\right| / 2: v(-1)=1, v(1)=-1, \text { and } v \text { solution of }(1.2)\right\} \text {. }
$$

The optimal value of this problem is $1 / 2$, and it is attained only for the minimizer $\tilde{u}$ of $(1.2)$ such that $\tilde{u}(x)=-x$ on $[-1,1]$. As a consequence, no other minimizer is an AML (and it can also be shown that this function $\tilde{u}$ is indeed an AML of (1.2)). 
Local minimizers are in some sense more meaningful than generic minimizers: when the supremand $f$ is smooth enough, such minimizers are solution of an Euler equation, and under suitable hypoteses it can be proven that they satisfy a maximum principle (we refer to [8]). For example, the model problem

$$
\min _{v \in W_{g}^{1, \infty}(\Omega)}\|D v\|_{L^{\infty}(\Omega)}=\min _{v \in W_{g}^{1, \infty}(\Omega)} \underset{x \in \Omega}{\operatorname{ess} . \sup }|D v(x)|
$$

admits a unique AML $u$ which is the unique viscosity solution of $-\Delta_{\infty}(u)=0$ in $\Omega$.

The aim of this paper is twofold: we first prove an approximation theorem and a lower-semicontinuity result for supremal functionals (Ths. 3.1 and 3.4) and then in the scalar case give two different existence theorems for AML (Ths. 4.1 and 4.7). The lower-semicontinuity result improves some known results (e.g. [7]) and is based on the $\Gamma$-convergence of the $L^{p}$ approximation technique for supremal functionals and on a suitable rearrangement technique. With this result, the existence of minimizers for problems of the type (1.1) follows by the direct method of the Calculus of Variations. Then the problem of the existence of AML arises.

Our first existence theorem (Th. 4.1) for AMLs is based on the same $L^{p}$ approximation and rearrangement arguments as in the proof of the lower-semicontinuity result. We notice that $L^{p}$ approximations have been already widely used for this problem in the literature $[2,8,19]$. Here we exploit the $\Gamma$-convergence character of this approximation (see Th. 3.1): this allows us to give a semicontinuity result under weak assumptions and also to distinguish which sequences of approximate minimizers converge to an AML and which a priori do not.

The second existence theorem (Th. 4.7) is based on a Perron-like method. It shows that the concept of AML is very natural for supremal functional and it gives an intrinsic proof (without approximation) for their existence. The method we use was introduced in [4] in the proof of the existence of an AML for the model problem (1.4), and it has been recently adapted to the metric setting [20].

The paper is organized as follows: Section 2 contains preliminary results and definitions which shall be used through the paper. The $L^{p}$ approximation (Th. 3.1) and the lower semicontinuity result (Th. 3.4) are stated and proven in Section 3. Finally, the two existence theorems for AMLs are stated and proven in Section 4.

\section{Preliminary Results}

\section{1. $\Gamma$-convergence}

Let $X$ be a metric space, a sequence of functionals $F_{n}: X \rightarrow \overline{\mathbb{R}}$ is said to $\Gamma$-converge to $F$ at $x$ if

$$
\Gamma-\lim \inf F_{n}(x)=\Gamma-\lim \sup F_{n}(x)
$$

where

$$
\left\{\begin{array}{l}
\Gamma-\lim \inf F_{n}(x)=\inf \left\{\liminf F_{n}\left(x_{n}\right): \quad x_{n} \rightarrow x\right\} \\
\Gamma-\lim \sup F_{n}(x)=\inf \left\{\limsup F_{n}\left(x_{n}\right): \quad x_{n} \rightarrow x\right\}
\end{array}\right.
$$

The $\Gamma$-convergence was introduced in [16], for an introduction to this theory we refer to [14]. The following classical theorem reports the characterizing properties of the $\Gamma$-convergence.

Theorem 2.1. Assume that $F_{n} \stackrel{\Gamma}{\rightarrow} F$ then $F$ is lower semicontinuous on $X$. Moreover if $F_{n}$ are equi-coercive on $X$ then $F$ is coercive too. In this last case, the following holds:

(1) the sequence $\left(\inf _{X} F_{n}\right)_{n}$ converges to the minimum of $F$ on $X$;

(2) if $x_{n}$ is such that $F_{n}\left(x_{n}\right) \leq \inf _{X} F_{n}+\varepsilon_{n}, \varepsilon_{n} \rightarrow 0$ and $x_{n_{k}} \rightarrow x$ for some subsequence $\left(x_{n_{k}}\right)_{k}$ of $\left(x_{n}\right)_{n}$ then $F(x)=\min _{X} F$.

\subsection{A lemma on Young measures}

The Young measures are one of the basic tools of the Calculus of Variations (e.g. [21,22]). The following proposition states a property of these object we shall need (see [10] for a proof). 
Proposition 2.2. Suppose that a sequence of maps $\left(z_{k}\right)_{k}$ generate a Young measure $\nu$. Let $f$ be a normal integrand and assume that the negative part $\left(f^{-}\left(., z_{k}(.)\right)\right)_{k}$ is weakly relatively compact in $L^{1}(E)$. Then

$$
\liminf _{k \rightarrow \infty} \int_{E} f\left(x, z_{k}(x)\right) \mathrm{d} x \geq \int_{E} \int_{\mathbb{R}^{N}} f(x, \lambda) \mathrm{d} \nu_{x}(\lambda) \mathrm{d} x .
$$

We will use the following application of the previous proposition: let $f: \Omega \times \mathbb{R}^{M} \times \mathbb{R}^{M \times N} \rightarrow \mathbb{R}$ be a non-negative function which is Borel measurable with respect to all variables and such that $f(x, \cdot, \cdot)$ is lower semicontinuous for almost every $x$ in $\Omega$ (then a normal integrand). Suppose that $u_{j} \rightarrow u$ in $W^{1, p}\left(\Omega, \mathbb{R}^{M}\right)$ and that $\left(D u_{j}\right)_{j}$ generates the Young measures $\nu$ (such a Young measure is usually called a $W^{1, p}$ gradient Young measure, see [24]). Then the couple $\left(u_{j}, D u_{j}\right)$ generates the Young measure $x \rightarrow \delta_{u(x)} \otimes \nu_{x}$, and by the previous proposition and remark

$$
\liminf _{j \rightarrow \infty} \int_{\Omega} f\left(x, u_{j}(x), D u_{j}(x)\right) \mathrm{d} x \geq \int_{\Omega} \int_{\mathbb{R}^{M \times N}} f(x, u(x), \lambda) d \nu_{x}(\lambda) \mathrm{d} x .
$$

\subsection{Level convexity and Morrey quasiconvex functions}

We now introduce briefly the notions of level-convexity and Morrey-quasiconvexity which are strongly related to one of the main hypothesis of our results, namely that the supremand $f$ satisfies a generalized Jensen inequality in the third variable (see (3.1) in Th. 3.1).

Definition 2.3. Let $f: \mathbb{R}^{M \times N} \rightarrow \overline{\mathbb{R}}$ is a level convex function if all its sublevel sets are convex, that is if $E_{\gamma}=\left\{x \in \mathbb{R}^{M \times N}: f(x) \leq \gamma\right\}$ is convex for every $\gamma \in \mathbb{R}$.

If $f: \mathbb{R}^{M \times N} \rightarrow \overline{\mathbb{R}}$ is lower semicontinuous and level convex, then it satisfies the following generalized Jensen inequality

$$
f\left(\int_{\mathbb{R}^{M \times N}} \phi(A) \mathrm{d} \mu(A)\right) \leq \mu-\operatorname{ess.sup}_{A \in \mathbb{R}^{M \times N}} f(\phi(A))
$$

for all probability measures $\mu$ on $\mathbb{R}^{M \times N}$ and all $\phi \in L_{\mu}^{1}\left(\mathbb{R}^{M \times N} ; \mathbb{R}^{M \times N}\right.$ ) (see Th. 1.2 in [7] for a proof, see also [6]).

A second class of functions (introduced and studied in [7]) is the following:

Definition 2.4. $f: \mathbb{R}^{M \times N} \rightarrow \mathbb{R}$ is (strong) Morrey-quasiconvex if for any $\varepsilon>0$, for any $A \in \mathbb{R}^{M \times N}$ and any $K>0$ there exists a $\delta=\delta(\varepsilon, K, A)>0$ such that if $\varphi \in W^{1, \infty}\left(Y, \mathbb{R}^{M}\right)$ satisfies

$$
\|D \varphi\|_{L^{\infty}(Y)} \leq K, \max _{\partial Y}|\varphi(x)| \leq \delta
$$

where $Y=] 0,1\left[^{N}\right.$, then

$$
f(A) \leq \underset{Y}{\operatorname{ess} . \sup } f(A+D \varphi) .
$$

It is not clear (and this was already pointed out in [7]) whether this last class of functions can be characterized in terms of some Jensen inequality. However thanks to the results of [7] this class of functions should be the natural setting for the semicontinuity of supremal functionals in the vectorial case $M>1$.

\section{3. $\Gamma$-CONVERGENCE AND A SEMICONTINUITY RESUlT}

In this part, we first prove a $\Gamma$-convergence result and then derive from this result a lower semicontinuity for supremal functionals.

The following theorem is one of the main results of the paper. We stress the variational character of the $L^{p}$ approximation, since it is the essential tool for the proof of Theorem 4.1 in Section 4.1. Notice that Theorem 3.1 is stated under a strong coercivity assumption, namely superlinearity of the integrand, that we shall drop in the lower semicontinuity result (Th. 3.4). 
Some of the arguments in the proof of this theorem are now rather classical (see $[8,9])$, however we make here a sharp use of the generalized Jensen inequality (3.1).

Theorem 3.1. Let $f: \Omega \times \mathbb{R}^{M} \times \mathbb{R}^{M \times N} \rightarrow[0,+\infty[$ be a normal integrand. Assume that $f$ satisfies the following generalized Jensen inequality

$$
\forall(x, s), \quad f\left(x, s, \int_{\mathbb{R}^{M \times N}} A d \nu_{x}(A)\right) \leq \nu_{x}-\underset{A \in \mathbb{R}^{M \times N}}{\operatorname{ess} \sup _{1}} f(x, s, A)
$$

whenever $\left(\nu_{x}\right)_{x \in \Omega}$ is an $W^{1, p}$ gradient Young measure for all $p \in(1, \infty)$. Assume further that $f$ has linear growth in its third varible, i.e. there exists a positive constant $c$ such that

$$
\forall(x, s, A) \quad f(x, s, A) \geq c\|A\|
$$

For any $p>N$, we define the functional $F_{p}: \mathrm{C}\left(\bar{\Omega}, \mathbb{R}^{M}\right) \rightarrow[0,+\infty]$ by

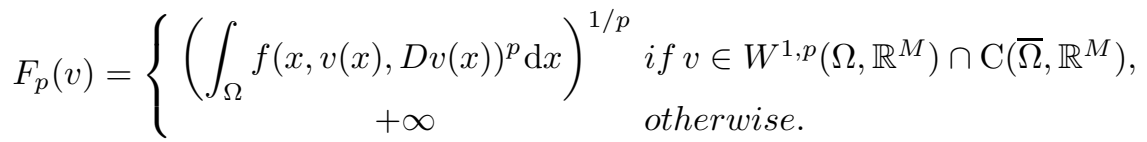

Then the family $\left(F_{p}\right)_{p>N} \Gamma$-converges to the functional $F$ given by

$$
F(v):= \begin{cases}\|f(., v(.), D v(.))\|_{L^{\infty}(\Omega)} & \text { if } v \in W^{1, \infty}\left(\Omega, \mathbb{R}^{M}\right) \cap \mathrm{C}\left(\bar{\Omega}, \mathbb{R}^{M}\right), \\ +\infty & \text { elsewhere }\end{cases}
$$

in $\mathrm{C}\left(\bar{\Omega}, \mathbb{R}^{M}\right)$ as $p$ goes to $+\infty$.

Proof. We first notice that for any $v$ in $\mathrm{C}\left(\bar{\Omega} ; \mathbb{R}^{M}\right)$, one has

$$
\limsup _{p \rightarrow \infty} F_{p}(v) \leq F(v)
$$

Indeed, if $F(v)=+\infty$, there is nothing to prove, and if $F(v)<+\infty$, then $f(\cdot, v(\cdot), D v(\cdot))$ belongs to $L^{p}(\Omega)$ for every $p \geq 1$ and

$$
\lim _{p \rightarrow \infty}\|f(\cdot, v(\cdot), D v(\cdot))\|_{L^{p}(\Omega)}=\|f(\cdot, v(\cdot), D v(\cdot))\|_{L^{\infty}(\Omega)},
$$

so that (3.4) follows from (3.3). As a consequence, $\Gamma-\lim \sup F_{p} \leq F$.

It remains to prove that $\Gamma-\liminf F_{p} \geq F$. Let $\left(v_{p}\right)_{p>N}$ be a family in $\mathrm{C}\left(\bar{\Omega}, \mathbb{R}^{M}\right)$ converging uniformly on $\Omega$ to some function $v$, we must check that

$$
\liminf _{p \rightarrow \infty} F_{p}\left(v_{p}\right) \geq F(v)
$$

We may assume without loss of generality that there exists $C$ in $\mathbb{R}$ such that $F_{p}\left(v_{p}\right) \leq C$ for any $p>N$. Then for any real numbers $p, q$ with $p>q>N$, one has

$$
\begin{aligned}
\left(\int_{\Omega}\left|D v_{p}(x)\right|^{q} \mathrm{~d} x\right)^{1 / q} & \leq \frac{1}{c}\left(\int_{\Omega} f\left(x, v_{p}(x), D v_{p}(x)\right)^{q} \mathrm{~d} x\right)^{1 / q} \\
& \leq \frac{1}{c}\left[|\Omega|^{1-q / p} F_{p}\left(v_{p}\right)^{q}\right]^{1 / q} \leq \frac{C}{c}|\Omega|^{1 / q-1 / p} .
\end{aligned}
$$


Thus $\left(D v_{p}\right)_{p>q}$ is bounded in $L^{q}\left(\Omega, \mathbb{R}^{N \times M}\right)$ for all $q>N$, so that $v_{p} \rightarrow v w-W^{1, q}$ for all $q>N$. In particular there is a $W^{1, p}$-gradient Young measure $\left\{\nu_{x}\right\}_{x \in \Omega}$ generated by $\left(D v_{p}\right)_{p>N}$ that satisfies

$$
D v(x)=\int_{\mathbb{R}^{M \times N}} A \mathrm{~d} \nu_{x}(A)
$$

for almost every $x$ in $\Omega$. We infer from the discussion following Proposition 2.2 that for any fixed $q>r>N$ one has

$$
\begin{aligned}
\liminf _{p \rightarrow \infty} F_{q}\left(v_{p}\right) & =\liminf _{p \rightarrow \infty}\left(\int_{\Omega} f\left(x, v_{p}(x), D v_{p}(x)\right)^{q} \mathrm{~d} x\right)^{1 / q} \\
& \geq\left[\int_{\Omega} \int_{\mathbb{R}^{M \times N}} f(x, v(x), A)^{q} d \nu_{x}(A) \mathrm{d} x\right]^{1 / q} \\
& \geq\left[\int_{\Omega}\left(\int_{\mathbb{R}^{M \times N}} f(x, v(x), A)^{r} d \nu_{x}(A)\right)^{q / r} \mathrm{~d} x\right]^{1 / q},
\end{aligned}
$$

where the last inequality follows from the convexity of $t \mapsto t^{q / r}$ on $\mathbb{R}_{+}$. As $q \rightarrow \infty$ we get

$$
\liminf _{q \rightarrow \infty} \liminf _{p \rightarrow \infty} F_{q}\left(v_{p}\right) \geq \underset{x \in \Omega}{\operatorname{ess.sup}}\left[\left(\int_{\mathbb{R}^{M \times N}} f(x, v(x), A)^{r} \mathrm{~d} \nu_{x}(A)\right)^{1 / r}\right] .
$$

Letting now $r$ tend to $+\infty$ yields

$$
\liminf _{q \rightarrow \infty} \liminf _{p \rightarrow \infty} F_{q}\left(v_{p}\right) \geq \underset{x \in \Omega}{\operatorname{ess.sup}}\left[\nu_{x}-\underset{A \in \mathbb{R}^{M \times N}}{\operatorname{ess.sup}} f(x, v(x), A)\right] .
$$

We now apply the extended Jensen inequality (3.1) with the gradient Young measures $\left(\nu_{x}\right)_{x \in \Omega}$ to obtain

$$
f(x, v(x), D v(x))=f\left(x, v(x), \int_{\mathbb{R}^{M \times N}} A \mathrm{~d} \nu_{x}\right) \leq \nu_{x}-\underset{A \in \mathbb{R}^{M \times N}}{\operatorname{ess.sup}} f(x, v(x), A)
$$

for almost every $x$ in $\Omega$. Hence we infer

$$
\begin{aligned}
\underset{\Omega}{\operatorname{ess.sup} f(x, v(x), D v(x))} & \leq \underset{\Omega}{\operatorname{ess.sup}}\left[\nu_{x}-\underset{A \in \mathbb{R}^{M \times N}}{\operatorname{ess.sup}} f(x, v(x), A)\right] \\
& \leq \liminf _{q \rightarrow \infty} \liminf _{p \rightarrow \infty} F_{q}\left(v_{p}\right) \\
& \leq \liminf _{q \rightarrow \infty} \liminf _{p \rightarrow \infty}|\Omega|^{1 / q-1 / p} F_{p}\left(v_{p}\right) .
\end{aligned}
$$

The right hand side of this inequality is equal to $\liminf _{p \rightarrow \infty} F_{p}\left(v_{p}\right)$, while the left hand side is equal to $F(v)$ since $v \in W^{1, \infty}\left(\Omega, \mathbb{R}^{M}\right) \cap \mathrm{C}\left(\bar{\Omega}, \mathbb{R}^{M}\right)$ (this last fact holding true by $v_{p} \rightarrow v$ uniformly and $\left\|D v_{p}\right\|_{q} \leq C^{\prime}$ for all $p>q$, see $(3.6))$.

Remark 3.2. Since no hypothesis is made on the regularity of the boundary of $\Omega$, the functions in $W^{1, \infty}\left(\Omega, \mathbb{R}^{M}\right)$ may not be continuous on $\bar{\Omega}$. One may thus think of studyingthe $\Gamma$-convergence of the family $\left(F_{p}\right)_{p}$ in $L^{1}\left(\Omega, \mathbb{R}^{M}\right)$ 
rather than in $\mathrm{C}\left(\bar{\Omega}, \mathbb{R}^{M}\right)$, where $F_{p}$ is now given by

$$
F_{p}(v)=\left\{\begin{aligned}
\left.\int_{\Omega} f(x, v(x), D v(x))^{p} \mathrm{~d} x\right)^{1 / p} & \text { if } u \in W^{1, p}\left(\Omega, \mathbb{R}^{M}\right), \\
+\infty & \text { if } u \in L^{1}\left(\Omega, \mathbb{R}^{M}\right) \backslash W^{1, p}\left(\Omega, \mathbb{R}^{M}\right) .
\end{aligned}\right.
$$

The same arguments as in the proof of Theorem 3.1 then yield the $\Gamma$-convergence of $F_{p}$.

Remark 3.3 (Fixing Boundary data). Notice that the previous result and proof also hold if we add boundary data by defining $F_{p}: \mathrm{C}\left(\bar{\Omega}, \mathbb{R}^{M}\right) \rightarrow \mathbb{R}_{+}$as follows

$$
F_{p}(v)=\left\{\begin{array}{cl}
\left.\int_{\Omega} f(x, v(x), D v(x))^{p} \mathrm{~d} x\right)^{1 / p} & \text { if } u \in W_{g}^{1, p}\left(\Omega, \mathbb{R}^{M}\right) \cap \mathrm{C}\left(\bar{\Omega}, \mathbb{R}^{M}\right), \\
+\infty & \text { otherwise }
\end{array}\right.
$$

where $g$ belongs to $W^{1, \infty}\left(\Omega, \mathbb{R}^{M}\right) \cap \mathrm{C}\left(\bar{\Omega}, \mathbb{R}^{M}\right)$.

We now infer from Theorem 3.1 the following semicontinuity result for supremal functionals. Notice that the growth assumptions made on the supremand $f$ are weakened in that result.

Theorem 3.4. Let $f: \Omega \times \mathbb{R}^{M} \times \mathbb{R}^{M \times N} \rightarrow \mathbb{R}$ be a normal integrand. Assume that $f$ satisfies the generalized Jensen inequality (3.1) and that is uniformly coercive in the third variable, i.e.

$$
\forall t \in \mathbb{R}, \quad \exists R \text {, s.t. } \forall(x, s), \quad\{A: f(x, s, A) \leq t\} \subset B(0, R) .
$$

Then the functional

$$
F(v):= \begin{cases}\underset{x \in \Omega}{\operatorname{ess.sup} f}(x, v(x), D v(x)) & \text { if } v \in W^{1, \infty}\left(\Omega ; \mathbb{R}^{M}\right) \cap \mathrm{C}\left(\bar{\Omega} ; \mathbb{R}^{M}\right), \\ +\infty & \text { elsewhere, }\end{cases}
$$

is lower semicontinuous in $\mathrm{C}\left(\bar{\Omega} ; \mathbb{R}^{M}\right)$ and then in $W^{1, \infty}\left(\Omega ; \mathbb{R}^{M}\right)$ with respect to the $w^{*}$-convergence.

Proof.

Step 1. We first reduce to the case where the supremand $f$ is non negative over $\Omega \times \mathbb{R}^{M} \times \mathbb{R}^{M \times N}$. To this end, we notice that it is sufficient to check that for any real number $\alpha$, the functional $F_{\alpha}:=\max \{F, \alpha\}$ is lower semicontinuous in $\mathrm{C}\left(\bar{\Omega} ; \mathbb{R}^{M}\right)$. Indeed if by contradiction we assume that there exists a sequence $\left(u_{n}\right)_{n}$ converging to $u$ uniformly such that

$$
\liminf _{n \rightarrow \infty} F\left(u_{n}\right)<F(u)
$$

then there exists $0<\varepsilon$ and a subsequence still denoted by $\left(u_{n}\right)$ such that $F\left(u_{n}\right) \leq F(u)-\varepsilon$ for $n$ large enough. Let $\alpha:=F(u)-\varepsilon$, then

$$
\liminf _{n \rightarrow \infty} F_{\alpha}\left(u_{n}\right) \leq F(u)-\varepsilon<F(u)=F_{\alpha}(u),
$$

which contradicts the lower-semicontinuity of $F_{\alpha}$.

Notice that the functional $F_{\alpha}$ is associated to the supremand $\max \{f, \alpha\}$, and that this supremand obviously satisfies (3.7) and (3.1) whenever $f$ does. We may thus assume that the supremand $f$ is bounded from below by some real constant $\alpha$. Since the functional associated to the supremand $f-\alpha$ is $F-\alpha$, we may assume that $\alpha=0$. From now on, we thus assume that $f$ is non-negative on $\Omega \times \mathbb{R}^{M} \times \mathbb{R}^{M \times N}$, so that $F$ may also be rewritten as follows

$$
F(v):= \begin{cases}\|f(., v(.), D v(.))\|_{L^{\infty}(\Omega)} & \text { if } v \in W^{1, \infty}\left(\Omega ; \mathbb{R}^{M}\right) \cap \mathrm{C}\left(\bar{\Omega} ; \mathbb{R}^{M}\right), \\ +\infty & \text { elsewhere. }\end{cases}
$$


Step 2. We now prove that there exists an increasing continuous function $h:[-1,+\infty) \rightarrow[0,+\infty[$ such that for all $(x, s, A)$ one has $(h \circ f)(x, s, A) \geq\|A\|$. For any $r \geq 0$, we set

$$
H(r):=\inf \left\{f(x, s, A): x \in \Omega, s \in \mathbb{R}^{M},\|A\| \geq r\right\} .
$$

Then for all $(x, s, A)$ one has $f(x, s, A) \geq H(\|A\|)$. Moreover, we notice that $H$ is nondecreasing and that $H(r) \rightarrow+\infty$ as $r$ goes to infinity since $f$ is uniformly coercive. Therefore there exists an increasing sequence $\left(r_{n}\right)_{n \in \mathbb{N}}$ of real numbers such that: $r_{0}=0$, and $r_{n} \geq r_{n-1}+1$ as well as $H\left(r_{n}\right) \geq H\left(r_{n-1}\right)+1$ for any $n \geq 1$. We define $H^{*}$ to be the only piecewise affine function on $\mathbb{R}_{+}$with value $k+n-1$ at each point $r_{n}$ with $n \geq 1$ and with value -1 at $r_{0}=0$. Notice that $H^{*}$ is increasing, continuous, one-to-one from $\mathbb{R}_{+}$to $[-1, \infty)$ and that $H \geq H^{*}$. Let $h:[-1, \infty) \mapsto \mathbb{R}_{+}$be its inverse, then $h$ has the desired properties since

$\forall(x, s, A) \quad(h \circ f)(x, s, A) \geq(h \circ H)(\|A\|) \geq\left(h \circ H^{*}\right)(\|A\|)=\|A\|$.

Step 3. Since $h$ is non decreasing and lower semicontinuous continuous, one has

$$
\underset{x \in \Omega}{\operatorname{ess.sup}}(h \circ g(x))=h(\underset{x \in \Omega}{\operatorname{ess} . \sup } g(x))
$$

for all measurable function $g$ on $\Omega$. As a consequence, the functional associated to $h \circ f$ is simply $h \circ F$ (where, of course, we set $h(+\infty)=+\infty)$, and since $h$ is continuous the lower semicontinuity of $F$ is equivalent to that of $h \circ F$. We now conclude: the supremand $h \circ f$ satisfies the hypotheses of Theorem 3.1 so that the functional $h \circ F$ is a $\Gamma$-limit and is thus lower semicontinuous (see Prop. 6.8 in [14]).

As already said, the above result applies to any level-convex supremand which satisfies the uniform coercivity condition (3.7). Notice in particular that convex functions are level-convex and then satisfy (3.1).

Remark 3.5. In the scalar case $(M=1)$, the hypothesis (3.1) characterizes the level-convexity, so that levelconvexity seems to be the good assumption in this setting. In the vectorial case $(M>1)$, it is proven in [7] that under regularity assumptions on $f$ stronger than that of Corollary 3.4 (strong) Morrey quasiconvexity is a necessary condition for lower semicontinuity. It is still an open problem to investigate the relationship between inequality (3.1) and (strong) Morrey quasi-convexity.

Let's make a short comparison with the other semicontinuity theorems existing in literature:

- in [7] the authors prove that the functional (3.3) is lower semicontinuous with respect to the $w^{*}-W^{1, \infty}$ convergence under the following assumptions on the supremand $f$ :

(1) $f$ is (strong) Morrey quasiconvex (see Def. 2.4) in the last variable;

(2) there exists a function $\omega: \mathbb{R}_{+} \times \mathbb{R}_{+} \rightarrow \mathbb{R}_{+}$which is continuous in its first variable and non decreasing in the second such that

$$
\left|f\left(x_{1}, s_{1}, A\right)-f\left(x_{2}, s_{2}, A\right)\right| \leq \omega\left(\left|x_{1}-x_{2}\right|+\left|s_{1}-s_{2}\right|,|A|\right) .
$$

Notice that in this result, no coercivity assumption is made on the supremand $f$, whereas our coercivity assumption (3.7) is a cornerstone of our proof of Theorem 3.4. However, the hypothesis (2) above rules out supremands such as

$$
f(x, A):=\left\{\begin{array}{cl}
\frac{1}{|x|}|A| & \text { if } x \neq 0, \\
0 & \text { if } x=0,
\end{array}\right.
$$

to which our theorem applies (for $\Omega=B(0,1)$ for example). This difference is interesting only in the vectorial case since in the scalar case only level convexity is assumed in [7]; 
- in [18] the authors consider a Serrin-type semicontinuity theorem (i.e. without coercivity assumptions) in the scalar case for the functional (3.3). The main results states that if $f$ is level-convex and for all $K \subset \subset \Omega \times \mathbb{R} \times \mathbb{R}^{N}$ there exists a modulus of continuity $\omega_{K}$ such that

$$
\left|f\left(x_{1}, s_{1}, A\right)-f\left(x_{2}, s_{2}, A\right)\right| \leq \omega_{K}\left(\left|x_{1}-x_{2}\right|+\left|s_{1}-s_{2}\right|\right),
$$

then (3.3) is lower semicontinuous with respect to the convergence defined by: $u_{n} \rightarrow u$ if and only if $u_{n}, u \in W^{1, \infty}$ and $u_{n} \rightarrow u$ uniformly;

- the semicontinuity for supremal functionals whose supremand do not depend on the gradient is studied in [1].

\section{EXISTENCE OF LOCAL MINIMIZERS}

We now turn to the existence of local minimizers for the variational problem $P(g, \Omega)$ given by

$P(g, \Omega)$

$$
\inf \left\{F(v, \Omega): v \in W^{1, \infty}(\Omega) \cap \mathrm{C}(\bar{\Omega}), v=g \text { on } \partial \Omega\right\}
$$

where $g$ is in $W^{1, \infty}(\Omega) \cap \mathrm{C}(\bar{\Omega})$ and $F$ is the supremal functional given by

$$
F(v, V):= \begin{cases}\underset{x \in V}{\operatorname{ess} . \sup f} f(x, v(x), D v(x)) & \text { if } v \in W^{1, \infty}(V) \cap \mathrm{C}(\bar{V}), \\ +\infty & \text { if } v \in \mathrm{C}(\bar{V}) \backslash W^{1, \infty}(V),\end{cases}
$$

where $V$ is an open subset of $\Omega$. Notice that in this last part, we restrict ourselves to the scalar case $M=1$. Under the assumptions of Theorem 3.4, the functional $F$ satisfies the following property:

$$
F(\cdot, \Omega) \text { is l.s.c. and coercive on } \mathrm{C}_{g}(\bar{\Omega}):=\{v \in \mathrm{C}(\bar{\Omega}): v=g \text { on } \partial \Omega\} \text {. }
$$

In $\left(H_{0}\right)$, coercive means that the sublevel sets $\{F \leq t\}$ (for $t$ in $\mathbb{R}$ ) are relatively compact in $\mathrm{C}_{g}(\bar{\Omega})$ for the topology of the uniform convergence. Under hypothesis $\left(H_{0}\right)$, problem $P(g, \Omega)$ admits at least one solution. We shall denote by $S(P(g, \Omega))$ the set of optimal solutions to $P(g, \Omega)$. With these notations, a function $u$ is an AML of $P(g, \Omega)$ if and only if $u$ belongs to $S(P(g, \Omega))$ and to $S(P(u, V))$ for any open subset $V$ of $\Omega$. In this section, we show that, under mild assumptions on the supremand $f$ or on the supremal funtional $F$, at least one of these solutions is a local solution of $P(g, \Omega)$. This result will be achieved in two different ways, and under two different sets of hypotheses.

\subsection{Existence by approximation}

In this section, we use the $L^{p}$ approximation method to prove the existence of an AML. The proof mainly relies on Proposition 4.3 which is very similar to that of Lemma 2.4 in [8] and is given here for the sake of completeness. For this method to work, some continuity assumption on the supremand $f$ is necessary in addition to the hypotheses of Theorem 3.4.

Theorem 4.1. Let $f: \Omega \times \mathbb{R} \times \mathbb{R}^{N} \rightarrow \mathbb{R}$ be a normal integrand continuous in the second variable. Assume that $f$ is uniformly coercive in the third variable (see (3.7)), that it satisfies the generalized Jensen inequality (3.1) and that $f$ is bounded from below by a constant $\alpha$, then problem $P(g, \Omega)$ admits at least one $A M L$.

The same rearrangement technique used for the semicontinuity allows to reduce the problem to the case in which $f$ is non-negative and has linear growth at infinity. The approximating functionals we consider are that introduced in Remark 3.3 and given by 


$$
F_{p}(v, V)=\left\{\begin{array}{cl}
\left(\int_{V} f(x, v(x), D v(x))^{p} \mathrm{~d} x\right)^{1 / p} & \text { if } v \in W^{1, p}(V) \cap \mathrm{C}(\bar{V}), \\
+\infty & \text { if } v \in \mathrm{C}(\bar{V}) \backslash W^{1, p}(V)
\end{array}\right.
$$

where $V$ is an open subset of $\Omega$. We now notice that the minima and approximate minimizers converge.

Proposition 4.2. The family of functionals $\left(F_{p}(., \Omega)\right)_{p \geq N+1}$ is equicoercive in $\mathrm{C}_{g}(\bar{\Omega})$ with respect to the uniform convergence of functions. Let $\varepsilon_{p}$ be a sequence of positive numbers such that $\varepsilon_{p} \rightarrow 0$ and let $u_{\infty}$ be a cluster point in $\mathrm{C}_{g}(\bar{\Omega})$ of a sequence $u_{p}$ such that $F_{p}\left(u_{p}, \Omega\right) \leq \inf F_{p}(., \Omega)+\varepsilon_{p}$ for all $p$. Then $u_{\infty}$ is a minimizer for problem $P(g, \Omega)$ (in particular $\left.u_{\infty} \in W^{1, \infty}\right)$.

Proof. The equicoercivity follows from the inequality (3.6) and of the compact imbeddings of Sobolev spaces. Indeed as we are working with fixed boundary data no regularity assumption is needed on the boundary of $\Omega$ (see for example [13] first remark on p. 26). The second part of the proposition follows from Theorem 3.1, Remark 3.3 and Theorem 2.1.

The problem is now to understand if the minimizers obtained in Proposition 4.2 are AML or not. In the next theorem and in the following example we will see that a quantitative argument is needed in order to guarantee that a sequence of approximate minimizers tends to an AML. This is due to the fact that the functionals we are considering are only subadditive with respect to the union of sets instead of being additive as the usual functionals of the Calculus of Variations.

Proposition 4.3. Let $\left(u_{p}\right)_{p}$ be a sequence of functions such that $F_{p}^{p}\left(u_{p}, \Omega\right) \leq \inf F_{p}^{p}(., \Omega)+\varepsilon_{p}$ where the sequence $\left(\varepsilon_{p}\right)_{p}$ is chosen so that $\lim _{p \rightarrow \infty} \sqrt[p]{\varepsilon_{p}}=0$. Let $\left(u_{p_{i}}\right)_{i}$ be a subsequence of $\left(u_{p}\right)_{p}$ converging uniformly on $\Omega$ to some function $u_{\infty}$.

Then $u_{\infty}$ is an $A M L$ for problem $P(g, \Omega)$.

Proof. From Proposition 4.2 we already know that $u_{\infty}$ is a minimizer of problem $P(g, \Omega)$. Let $V \subset \Omega$ be open and let $\varphi \in W^{1, \infty}(V) \cap \mathrm{C}_{0}(\bar{V})$, so that we have $u_{\infty}+\varphi=u_{\infty}$ on $\partial V$.

We first notice that we can assume that $\varphi>0$ in $V$. Indeed, if this is not the case

$$
F\left(u_{\infty}+\varphi, V\right)=\max \left\{F\left(u_{\infty}+\varphi,\{\varphi>0\}\right), F\left(u_{\infty}+\varphi,\{\varphi<0\}\right), F\left(u_{\infty}+\varphi,\{\varphi=0\}\right)\right\}
$$

and then our next arguments will apply to the first two elements of the set on the right side of (4.1) while the third does not really matter in the comparison with $F\left(u_{\infty}, V\right)$. Fix $\delta>0$ such that $|\{x \in V: \varphi>\delta\}|>0$. Since $\left(u_{p}\right)_{p}$ converges uniformly to $u_{\infty}$, there exists $p_{\delta}$ such that for every $p \geq p_{\delta}$ we have $u_{p}+\frac{\delta}{2}>u_{\infty}>u_{p}-\frac{\delta}{2}$ in $\Omega$. In particular $V_{\delta}:=\{x \in V: \varphi>\delta\}$ is included in $V_{p, \delta}:=\left\{x \in V: u_{p}+\frac{\delta}{2}<u_{\infty}+\varphi\right\}$ for such real numbers $p$. Notice that $V_{p, \delta}$ is an open set and that on its boundary one has $u_{p}=-\frac{\delta}{2}+u_{\infty}+\varphi$. Thus, for every $p \geq p_{\delta}$, we have

and then

$$
F_{p}^{p}\left(u_{p}, V_{p, \delta}\right) \leq F_{p}^{p}\left(u_{\infty}+\varphi-\frac{\delta}{2}, V_{p, \delta}\right)+\varepsilon_{p}
$$

$$
F_{p}\left(u_{p}, V_{\delta}\right) \leq F_{p}\left(u_{\infty}+\varphi-\frac{\delta}{2}, V_{p, \delta}\right)+\sqrt[p]{\epsilon}{ }_{p} \leq F\left(u_{\infty}+\varphi-\frac{\delta}{2}, V\right)|V|^{1 / p}+\sqrt[p]{\epsilon}{ }_{p}
$$

By Remark 3.3,

$$
F\left(u_{\infty}, V_{\delta}\right) \leq \liminf _{p \rightarrow+\infty} F_{p}\left(u_{p}, V_{\delta}\right) \leq F\left(u_{\infty}+\varphi-\frac{\delta}{2}, V\right) .
$$

Passing to the limit for $\delta \rightarrow 0^{+}$and using the continuity of $f$ with respect to the second variable, we obtain

$$
F\left(u_{\infty}, V\right) \leq F\left(u_{\infty}+\varphi, V\right)
$$

which concludes the proof. 
The next example shows that the control on the behaviour of $\varepsilon_{p}$ when $p \rightarrow 0$ is necessary.

Example 4.4. Let $v_{\infty}$ be a minimizer of $F$ which is not an AML. Then as $F_{p}\left(v_{\infty}\right) \rightarrow F\left(v_{\infty}\right)$ the sequence $v_{p}=v_{\infty}$ for all $p$ is a sequence of approximate minimizers, indeed if we denote by $M_{p}$ the infimum of $F_{p}$ and by $M$ the infimum of $F$ we have that

$$
\left|F_{p}\left(v_{\infty}\right)-M_{p}\right| \leq\left|F_{p}\left(v_{\infty}\right)-M\right|+\left|M-M_{p}\right|
$$

and the right hand term tends to 0 . On the other hand it is clear that the sequence converges to $v_{\infty}$ which is a minimizer but not an AML by assumption.

Corollary 4.5. If for any $p$ the functional $F_{p}$ admits a least one minimizer and if those minimizers converge (up to subsequences) as $p \rightarrow \infty$ to some function $u$, then $u$ is an $A M L$.

Remark 4.6. The need to specify the behaviour of $\varepsilon_{p} \rightarrow 0$ in Proposition 4.3 reflects the instability when $p \rightarrow \infty$ of the so called fundamental estimates which are the foundamental tools to prove the convergence of local minimizers (see [15]).

\subsection{An intrinsic approach to existence of AML}

Here we show the existence of an AML without making use of the approximation process of the last section, but by applying a Perron-like method. This proof relies on rather natural hypotheses on the functional $F$ and on a connectedness assumption on the optimal set of problem $P(g, \Omega)$. Let us detail the assumptions of Theorem 4.7 below. We first need to make more precise hypothesis $\left(H_{0}\right)$ and set

$$
\begin{aligned}
& F(., V) \text { is l.s.c. and coercive on } \mathrm{C}_{w}(\bar{V}):=\{v \in \mathrm{C}(\bar{V}): v=w \text { on } \partial V\} \\
& \text { for any open subset } V \text { of } \Omega \text { and } w \in W^{1, \infty}(V) \cap \mathrm{C}(\bar{V}) \text {. }
\end{aligned}
$$

Of course $F$ satisfies $\left(H_{1}\right)$ as soon as the hypotheses of Theorem 3.4 are fullfilled. We shall also need the following additional hypothesis on the structure of the optimal sets of $S(P(g, \Omega))$ :

$$
\text { for any open subset } V \text { of } \Omega, w \in W^{1, \infty}(V) \cap \mathrm{C}(\bar{V}) \text { and } y \in V \text {, }
$$$$
\text { the image set }\{u(y): u \in S(P(w, V))\} \text { is connected. }
$$

The hypothesis $\left(H_{2}\right)$ is automatically satisfied if the functional $F$ is for example convex, or level-convex in the variable $v$ (which means that the supremand $f$ is level-convex in $(s, A)$ ). Notice that in the case when the supremand $(x, s, A) \mapsto f(x, s, A)$ does not depend on the variable $s$, the hypothesis (3.1) of Theorem 3.4 implies that $f$ is level-convex in $A$, so that $\left(H_{2}\right)$ is fullfilled.

The main result of this section thus reads:

Theorem 4.7. Assume that the functional $F$ is such that $\left(H_{1}\right)$ and $\left(H_{2}\right)$ hold. Then the problem $P(g, \Omega)$ admits at least one $A M L$.

The rest of this section is devoted to the proof of Theorem 4.7.

Lemma 4.8. If $u$ and $v$ are two solutions of $P(g, \Omega)$, then $\max (u, v)$ and $\min (u, v)$ are also solutions of $P(g, \Omega)$.

Proof. It is sufficient to notice that if $V$ denotes the open set where $u>v$, then

$$
\begin{aligned}
F(\max (u, v), \Omega) & =\max (\underset{V}{\operatorname{ess.sup} f} f(x, u(x), D u(x)) ; \underset{\Omega \backslash V}{\operatorname{ess.sup}} f(x, v(x), D v(x))) \\
& \leq \max (F(u, \Omega), F(v, \Omega))=\inf (P(g, \Omega))
\end{aligned}
$$

so that $\max (u, v)$ belongs to $S(P(g, \Omega))$. The proof is analogous for $\min (u, v)$. 
Proposition 4.9. Assume that $\left(H_{1}\right)$ holds. The functions $S^{-}(g, \Omega): x \mapsto \inf _{u \in S(P(g, \Omega))} u(x)$ and $S^{+}(g, \Omega)$ : $x \mapsto \sup _{u \in S(P(g, \Omega))} u(x)$ are solutions of $P(g, \Omega)$.

Proof. We only prove that $S^{+}(g, \Omega)$ belongs to $S(P(g, \Omega))$. Let $\left(x_{n}\right)_{n \in \mathbb{N}}$ be a denumerable family which is dense in $\Omega$. For any integers $n$ and $i \leq n$, there exists a solution $u_{n}^{i}$ of $P(g, \Omega)$ such that

$$
u_{n}^{i}\left(x_{i}\right) \geq S^{+}(g, \Omega)\left(x_{i}\right)-\frac{1}{n} .
$$

Then Lemma 4.8 implies that the function $u_{n}:=\max \left(u_{n}^{1}, \ldots, u_{n}^{n}\right)$ is a solution of $P(g, \Omega)$. It results that

$$
\forall i \leq n \quad S^{+}(g, \Omega)\left(x_{i}\right) \geq u_{n}\left(x_{i}\right) \geq S^{+}(g, \Omega)\left(x_{i}\right)-\frac{1}{n}
$$

so that $u_{n} \rightarrow S^{+}(g, \Omega)$ on a dense subset of $\Omega$. This implies that $u_{n} \rightarrow S^{+}(g, \Omega)$ uniformly on $\Omega$ since, by coercivity, the sequence $\left(u_{n}\right)_{n}$ of minimizers is relatively compact in $\mathrm{C}(\bar{\Omega})$. By lower semicontinuity of $F$, $S^{+}(g, \Omega)$ is a solution of $P(g, \Omega)$.

Definition 4.10. We say that $u \in \mathrm{C}(\bar{\Omega})$ is a local subsolution of $P(g, \Omega)$ if $u$ is a solution of $P(g, \Omega)$ and for all open subset $V$ of $\Omega, u \leq S^{+}(u, V)$ on $V$.

Analogously, $u \in \mathrm{C}(\bar{\Omega})$ is a local supersolution of $P(g, \Omega)$ if $u$ is a solution of $P(g, \Omega)$ and for all open subset $V$ of $\Omega, u \geq S^{-}(u, V)$ on $V$.

We shall denote $S^{+\operatorname{loc}}(P(g, \Omega))$ (resp. $\left.S^{-\operatorname{loc}}(P(g, \Omega))\right)$ the set of all local supersolutions (resp. subsolutions) of $P(g, \Omega)$.

Lemma 4.11. Assume that $\left(H_{1}\right)$ holds. The function $S^{+}(g, \Omega)$ is a local supersolution of $P(g, \Omega)$, and $S^{-}(g, \Omega)$ is a local subsolution of $P(g, \Omega)$.

Proof. It is sufficient to verify that $S^{+}(g, \Omega)$ is a local supersolution of $P(g, \Omega)$. Let $V$ be an open subset of $\Omega$, we define a function $u$ on $\Omega$ by

$$
u:=\left\{\begin{array}{cc}
\max \left\{S^{-}\left(S^{+}(g, \Omega), V\right) ; S^{+}(g, \Omega)\right\} & \text { on } V \\
S^{+}(g, \Omega) & \text { on } \Omega \backslash V .
\end{array}\right.
$$

Since $\left.F\left(S^{-}\left(S^{+}(g, \Omega), V\right), V\right)\right) \leq F\left(S^{+}(g, \Omega), V\right)$ and $u=S^{+}(g, \Omega)$ on $\Omega \backslash V, u$ is a solution of $P(g, \Omega)$. By definition, $u$ is thus lower than $S^{+}(g, \Omega)$ on $\Omega$, so that $S^{+}(g, \Omega) \geq S^{-}\left(S^{+}(g, \Omega), V\right)$ on $V$.

Remark 4.12. Arguing as in the proof of the above lemma, we also have the stronger property $S^{+}(g, \Omega) \geq$ $S^{+}\left(S^{+}(g, \Omega), V\right)$, for every open subset $V$ of $\Omega$.

Proposition 4.13. Assume that $\left(H_{1}\right)$ and $\left(H_{2}\right)$ hold. A function $u$ is an $A M L$ of $P(g, \Omega)$ if and only if it is both a local supersolution and a local subsolution of $P(g, \Omega)$.

Proof. We first notice that if $u$ is an AML of $P(g, \Omega)$, then for every open subset $V$ of $\Omega, u$ is a solution of $P(u, V)$ so by definition, $S^{-}(u, V) \leq u \leq S^{+}(u, V)$. As a consequence, $u$ is both a local supersolution and a local subsolution of $P(g, \Omega)$, which concludes the proof of the if part.

Now suppose that a function $u$ is both a local supersolution and a local subsolution of $P(g, \Omega)$. Then in particular it is a solution of $P(g, \Omega)$.

Let now $V$ be an open subset of $\Omega$, we must check that $u$ is a solution of $P(u, V)$. Let $\left(x_{n}\right)_{n \in \mathbb{N}}$ be a denumerable family which is dense in $V$. We construct by induction on $n$ a family $\left(u_{n}\right)_{n \in \mathbb{N}}$ of solutions of $P(u, V)$ such that for any integers $n$ and $i \leq n$ one has $u_{n}\left(x_{i}\right)=u\left(x_{i}\right)$. To this end, we notice that since $u$ is both a local supersolution and a local subsolution of $P(g, \Omega)$, we have $S^{-}(u, V) \leq u \leq S^{+}(u, V)$ on $V$. Then thanks to hypothesis $\left(H_{2}\right)$, there exists a solution $u_{1}$ of $P(u, V)$ such that $u_{1}\left(x_{1}\right)=u\left(x_{1}\right)$. Now we assume that $u_{n}$ has been constructed and we define $u_{n+1}$. To this end, we consider the problem $P\left(u, V_{n}\right)$ where 
$V_{n}:=V \backslash\left\{x_{1}, \ldots, x_{n}\right\}$ : since the function $u_{n}$ is a solution of $P(u, V)$ which is equal to $u$ on the set $\left\{x_{1}, \ldots, x_{n}\right\}$, we infer that it is an admissible function for $P\left(u, V_{n}\right)$. As a consequence, the value of the infimum of the problem $P\left(u, V_{n}\right)$ is equal to that of $P(u, V)$, and the optimal set $S\left(P\left(u, V_{n}\right)\right)$ is included in $S(P(u, V))$. Once again, since $u$ is both a local supersolution and a local subsolution of $P(g, \Omega)$, we have $S^{-}\left(u, V_{n}\right) \leq u \leq S^{+}\left(u, V_{n}\right)$ on $V_{n}$. Therefore, $\left(H_{2}\right)$ yields the existence of a solution $u_{n+1}$ of $P\left(u, V_{n}\right)$ which is equal to $u$ at the point $x_{n+1}$. This function $u_{n+1}$ has the desired properties: it is a solution of $P\left(u, V_{n}\right)$ and thus of $P(u, V)$, it is equal to $u$ at $x_{n+1}$, and since it is admissible for $P\left(u, V_{n}\right)$, it is also equal to $u$ on $\left\{x_{1}, \ldots, x_{n}\right\}$. As a family of solutions of $P(u, V)$, the family $\left(u_{n}\right)_{n \in \mathbb{N}}$ is equilipschitzian on $V$, and since $\left(x_{n}\right)_{n \in \mathbb{N}}$ is dense in $V$, it converges uniformly to $u$ on $\bar{V}$. We then infer from $\left(H_{1}\right)$ that $u$ is a solution of $P(u, V)$, which concludes the only if part of the proof.

Proposition 4.14. Assume that $\left(H_{1}\right)$ holds. The function $U^{+}: x \mapsto \sup \left\{u(x): u \in S^{-\operatorname{loc}}(P(g, \Omega))\right\}$ is both a local supersolution and a local subsolution of $P(g, \Omega)$.

Proof. The same argument as in the proof of Proposition 4.9 shows that the function $U^{+}$may be obtained as a uniform limit of local subsolutions of $P(g, \Omega)$, so it is a solution of $P(g, \Omega)$.

Let us check that $U^{+}$is a local subsolution of $P(g, \Omega)$. Let $V$ be an open subset of $\Omega$, and assume that for some $x_{0}$ in $V$, one has $U^{+}\left(x_{0}\right)>S^{+}\left(U^{+}, V\right)\left(x_{0}\right)$. Then by definition of $U^{+}$, there exists a local subsolution $u$ of $P(g, \Omega)$ such that $u\left(x_{0}\right)>S^{+}\left(U^{+}, V\right)\left(x_{0}\right)$. Since $u$ is lower than $U^{+}$, it is also lower than $S^{+}\left(U^{+}, V\right)$ on $\partial V$. Let us set $A:=\left\{x: u(x)>S^{+}\left(U^{+}, V\right)(x)\right\}$, then we must have $u \leq S^{+}(u, A)=S^{+}\left(S^{+}\left(U^{+}, V\right), A\right)$ on $A$ since $u$ is a local subsolution of $P(g, \Omega)$. By Remark 4.12 we have that $\bar{S}^{+}\left(S^{+}\left(U^{+}, V\right), A\right)$ is lower than $S^{+}\left(U^{+}, V\right)$ on $A$. As a consequence, we have $u \leq S^{+}\left(U^{+}, V\right)$ on $A$, which is a contradiction.

To check that $U^{+}$is a local supersolution of $P(g, \Omega)$, we have to prove that the set $A=\left\{x \in V: U^{+}(x)<\right.$ $\left.S^{-}\left(U^{+}, V\right)(x)\right\}$ is empty for any open subset $V$ of $\Omega$. This is equivalent to prove that the function $u$ defined by

$$
u:=\left\{\begin{array}{ccc}
S^{-}\left(U^{+}, V\right) & \text { on } & A \\
U^{+} & \text {on } & \Omega \backslash \bar{A}
\end{array}\right.
$$

is a local subsolution of $P(g, \Omega)$. Thus we fix an open subset $B$ of $\Omega$ and prove that $u \leq S^{+}(u, B)$ on $B$. Let us define $C:=B \cap\left\{u>S^{+}(u, B)\right\}$, we want $C$ to be empty. Assume the contrary, and consider $D:=B \cap\left\{U^{+}>\right.$ $\left.S^{+}(u, B)\right\}$; thus $C \cap(B \backslash D) \subset A$. If $D$ is empty then $C \subset A$ and so

$$
\begin{aligned}
u=S^{-}\left(U^{+}, V\right) & \leq S^{+}\left(S^{-}\left(U^{+}, V\right), C\right)=S^{+}(u, C)=S^{+}\left(S^{+}(u, B), C\right) \\
& \leq S^{+}(u, B)<u \quad \text { on } \mathrm{C} .
\end{aligned}
$$

The set $C$ being non empty, this is a contradiction, and we thus infer that $D$ is non empty. On the other hand, $U^{+}=S^{+}(u, B)$ on $\partial D$ by definition of $D$, hence as $U^{+}$is a subsolution we have

$$
U^{+} \leq S^{+}\left(S^{+}(u, B), D\right) \leq S^{+}(u, B) \quad \text { on } \mathrm{D}
$$

which contradicts the definition of $D$. As a consequence: since $U^{+}$is a subsolution, $D$ is empty, and so is $C$ and the proof is complete.

Theorem 4.7 is now a direct consequence of the above serie of results. Indeed, $U^{+}$is an AML thanks to Propositions 4.13 and 4.14 .

Remark 4.15. The same proof as in Proposition 4.14 yields that the function $U^{-}: x \mapsto \inf \{u(x): u \in$ $\left.S^{+ \text {loc }}(P(g, \Omega))\right\}$ is both a local supersolution and a local subsolution of $P(g, \Omega)$. It is thus also an AML of $P(g, \Omega)$, and one has that for every AML $u$ of $P(g, \Omega): U^{-} \leq u \leq U^{+}$.

Remark 4.16. Note that the hypothesis $\left(H_{2}\right)$ is only necessary in our proof of the caracterization of AMLs as local sub- and supersolutions (Prop. 4.13). 
Acknowledgements. The authors warmly thank the referees for the many usefull remarks and comments. Part of this work was done while the first author was on a postdoctoral position at the Centro de Modelamiento Matemático of the Universidad de Chile (Santiago de Chile). During the preparation of this paper the second author was partially supported by: the italian I.N.D.A.M. (Istituto Nazionale di Alta Matematica "Francesco Severi") through a senior scholarship and by the European Research Training Network "Homogenization and Multiple Scales" under contract HPRN-2000-00109.

\section{REFERENCES}

[1] E. Acerbi, G. Buttazzo and F. Prinari, On the class of functionals which can be represented by a supremum. J. Convex Anal. 9 (2002) 225-236.

[2] G. Aronsson, Minimization Problems for the Functional $\sup _{x} F\left(x, f(x), f^{\prime}(x)\right)$. Ark. Mat. 6 (1965) 33-53.

[3] G. Aronsson, Minimization Problems for the Functional $\sup _{x} F\left(x, f(x), f^{\prime}(x)\right)$. II. Ark. Mat. 6 (1966) 409-431.

[4] G. Aronsson, Extension of Functions satisfying Lipschitz conditions. Ark. Mat. 6 (1967) 551-561.

[5] G. Aronsson, Minimization Problems for the Functional $\sup _{x} F\left(x, f(x), f^{\prime}(x)\right)$. III. Ark. Mat. 7 (1969) 509-512.

[6] E.N. Barron, Viscosity solutions and analysis in $L^{\infty}$. Nonlinear Anal. Differential Equations Control. Montreal, QC (1998) 1-60. Kluwer Acad. Publ., Dordrecht, NATO Sci. Ser. C Math. Phys. Sci. 528 (1999).

[7] E.N. Barron, R.R. Jensen and C.Y. Wang, Lower Semicontinuity of $L^{\infty}$ functionals. Ann. Inst. H. Poincaré Anal. Non Linéaire 18 (2001) 495-517.

[8] E.N. Barron, R.R. Jensen and C.Y. Wang, The Euler equation and absolute minimizers of $L^{\infty}$ functionals. Arch. Rational Mech. Anal. 157 (2001) 255-283.

[9] T. Bhattacharya, E. DiBenedetto and J. Manfredi, Limits as $p \rightarrow \infty$ of $\Delta_{p} u_{p}=f$ and related extremal problems, Some topics in nonlinear PDEs. Turin (1989). Rend. Sem. Mat. Univ. Politec. Torino 1989, Special Issue (1991) 15-68.

[10] H. Berliocchi and J.M. Lasry, Intégrandes normales et mesures paramétrées en calcul des variations. Bull. Soc. Math. France 101 (1973) 129-184.

[11] M.G. Crandal and L.C. Evans, A remark on infinity harmonic functions, in Proc. of the USA-Chile Workshop on Nonlinear Analysis. Vina del Mar-Valparaiso (2000) 123-129. Electronic. Electron. J. Differential Equations Conf. 6. Southwest Texas State Univ., San Marcos, TX (2001).

[12] M.G. Crandal, L.C. Evans and R.F. Gariepy, Optimal Lipschitz extensions and the infinity Laplacian. Calc. Var. Partial Differential Equations 13 (2001) 123-139.

[13] B. Dacorogna, Direct methods in the calculus of variations. Springer-Verlag, Berlin, Appl. Math. Sci. 78 (1989).

[14] G. Dal Maso, An Introduction to Г-Convergence. Birkhauser, Basel, Progr. in Nonlinear Differential Equations Appl. 8 (1993).

[15] G. Dal Maso and L. Modica, A general theory of variational functionals. Topics in functional analysis (1980-81) 149-221. Quaderni, Scuola Norm. Sup. Pisa, Pisa (1981).

[16] E. De Giorgi and T. Franzoni, Su un tipo di convergenza variazionale. Atti Accad. Naz. Lincei Rend. Cl. Sci. Fis. Mat. Natur. (8) $\mathbf{5 8}$ (1975) 842-850.

[17] A. Garroni, V. Nesi and M. Ponsiglione, Dielectric Breakdown: Optimal bounds. Proc. Roy. Soc. London Sect. A 457 (2001) $2317-2335$.

[18] M. Gori and F. Maggi, On the lower semicontinuity of supremal functional. ESAIM: COCV 9 (2003) 135.

[19] R.R. Jensen, Uniqueness of Lipschitz Extensions: Minimizing the Sup Norm of the Gradient. Arch. Rational Mech. Anal. 123 (1993) 51-74.

[20] P. Juutinen, Absolutely Minimizing Lipschitz Extensions on a metric space. An. Ac. Sc. Fenn. Mathematica 27 (2002) 57-67.

[21] D. Kinderlehrer and P. Pedregal, Characterization of Young Measures Generated by Gradients. Arch. Rational Mech. Anal. 115 (1991) 329-365.

[22] D. Kinderlehrer and P. Pedregal, Gradient Young Measures Generated by Sequences in Sobolev Spaces. J. Geom. Anal. 4 (1994) 59-90.

[23] S. Muller, Variational models for microstructure and phase transitions. Calculus of variations and geometric evolution problems. Cetraro (1996) 85-210. Springer, Berlin, Lecture Notes in Math. 1713 (1999).

[24] P. Pedregal, Parametrized measures and variational principles. Birkhäuser Verlag, Basel, Progr. in Nonlinear Differential Equations Appl. 30 (1997). 